\title{
MicroRNA-93 Regulates Hypoxia-Induced Autophagy by Targeting ULK1
}

\author{
Wen Li, ${ }^{1,2}$ Yue Yang, ${ }^{3}$ Zhaoyu Ba, ${ }^{4}$ Shupeng $\mathrm{Li}^{5}{ }^{5} \mathrm{Hao} \mathrm{Chen},{ }^{5}$ Xiaoyan $\mathrm{Hou},{ }^{5}$ Linlin Ma, \\ Pengcheng He, ${ }^{7}$ Lei Jiang, ${ }^{7}$ Longxuan $\mathrm{Li}^{8}{ }^{8}$ Rongrong He, ${ }^{2}$ Liangqing Zhang, ${ }^{3}$ and Du Feng ${ }^{1,5}$
}

${ }^{1}$ Key Laboratory of Protein Modification and Degradation, School of Basic Medical Sciences, Affiliated Cancer Hospital \& Institute of Guangzhou Medical University, Guangzhou Medical University, Guangzhou 511436, China

${ }^{2}$ Anti-Stress and Health Research Center, College of Pharmacy, Jinan University, Guangzhou 510632, China

${ }^{3}$ Department of Anesthesiology, Guangdong Medical University, Zhanjiang 524001, China

${ }^{4}$ Department of Spine Surgery, Shanghai East Hospital, Tongji University School of Medicine, Tongji University, Shanghai 200120, China

${ }^{5}$ Institute of Neurology, Guangdong Key Laboratory of Age-Related Cardiac-Cerebral Vascular Disease, Affiliated Hospital of Guangdong Medical University, Zhanjiang, Guangdong 524001, China

${ }^{6}$ Department of Obstetrics and Gynecology, Beijing Hospital, National Center of Gerontology, Beijing 100730, China

${ }^{7}$ Department of Cardiology, Guangdong Cardiovascular Institute, Guangdong Provincial Key Laboratory of Coronary Heart Disease Prevention, Guangdong General Hospital, Guangdong Academy of Medical Sciences, Guangzhou, China

${ }^{8}$ Department of Neurology, Gongli Hospital, Pudong New Area, Shanghai 200135, China

Correspondence should be addressed to Rongrong He; rongronghe66@163.com, Liangqing Zhang; zhanglq1970@163.com, and Du Feng; feng_du@foxmail.com

Received 30 January 2017; Revised 13 July 2017; Accepted 8 August 2017; Published 3 October 2017

Academic Editor: Savita Khanna

Copyright ( 92017 Wen Li et al. This is an open access article distributed under the Creative Commons Attribution License, which permits unrestricted use, distribution, and reproduction in any medium, provided the original work is properly cited.

The expression of the core autophagy kinase, Unc51-like kinase 1 (ULK1), is regulated transcriptionally and translationally by starvation-induced autophagy. However, how ULK1 is regulated during hypoxia is not well understood. Previously, we showed that ULK1 expression is induced by hypoxia stress. Here, we report a new ULK1-modulating microRNA, miR-93; its transcription is negatively correlated with the translation of ULK1 under hypoxic condition. miR-93 targets ULK1 and reduces its protein levels under hypoxia condition. miR-93 also inhibits hypoxia-induced autophagy by preventing LC3-I to LC3-II transition and P62 degradation; these processes are reversed by the overexpression of an endogenous miR-93 inhibitor. Re-expression of ULK1 without miR-93 response elements restores the hypoxia-induced autophagy which is inhibited by miR-93. Finally, we detected the effects of miR-93 on cell viability and apoptosis in noncancer cell lines and cancer cells. We found that miR-93 sustains the viability of MEFs (mouse embryonic fibroblasts) and inhibits its apoptosis under hypoxia. Thus, we conclude that miR-93 is involved in hypoxia-induced autophagy by regulating ULK1. Our results provide a new angle to understand the complicated regulation of the key autophagy kinase ULK1 during different stress conditions.

\section{Introduction}

Autophagy is a regulated and highly conserved process that can develop double-membraned autophagosomes to degrade large protein aggregates and damaged organelles upon fusing with lysosomes [1, 2]. Autophagy can not only recycle intracellular energy resources when the cell is deficient in nutrients but also remove cytotoxic proteins and damaged organelles under a lot of stress conditions including pathogen invasion, hypoxia, and mitochondrial depolarization [3-5].

ULK1 is a mammalian homologue of yeast Atg1 [6, 7]. As an initiator of autophagy, ULK1 regulates autophagy through interaction of upstream mTOR and AMPK and then transduce signals to downstream mediators [8-13]. ULK1 protein level can be regulated by a variety of stimuli such as nutrientdepleted condition, energy deprivation, and hypoxia [14-16]. 
In the initial stage of nutrient-depletion condition, the elevated transcription of ULK1 is immediately induced, but after prolonged starvation, the ULK1 protein level is reduced because of the suppressed translation as well as the increased degradation of ULK1, which limits further autophagy induction [17-19]. ULK1 level is significantly increased in response to inhibition of mitochondrial respiratory complexes in cell treated with inhibitors of the mitochondrial complex I (rotenone), complex II (thenoyltrifluoroacetone/TTFA), or complex III (antimycin A or myxothiazol) [17]. Previously, we also found that ULK1 level is induced in cells subjected to $1 \%$ oxygen stress condition or by mitochondrial depolarized drug, FCCP, indicating that the ULK1 level is regulated at both transcriptional and translational levels and is more complicatedly controlled than previously thought $[15,16]$.

miRNAs are $17 \sim 22$ nucleotide, noncoding and singlestranded RNA molecules playing a role in a variety of pathophysiologic processes including apoptosis, cell proliferation, and differentiation [20-22]. It targets multiple genes via blocking mRNA translation. Silencing Dicer1, a major component of the miRNA-silencing machinery, increases the levels of the Atg protein and the formation of autophagosomes in cells [23]. Kap1, also known as TRIM28 (tripartite motif protein 28), acts as a scaffold for a multimolecular complex that silences transcription through the formation of heterochromatin $[24,25]$. Hematopoietic-restricted deletion of Kap1 fails to induce mitophagy (the process of removing damaged mitochondrial through autophagy)associated genes [26, 27] and retains mitochondria due to lack of repression of a subset of microRNAs, which target mitophagy transcripts. We also found that hypoxiaresponsive miR-137 regulates mitophagy by targeting two mitophagy receptors FUNDC1 and NIX. These findings indicate that miRNAs can regulate autophagy [28, 29]. Although several studies on microRNA regulation of ULK1 have been reported, those microRNAs do not respond to hypoxic environments [30-38].

miR-93 plays a variety of roles in regulating cellular homeostasis. For example, the expression of miR-93 causes hypersusceptibility to vesicular stomatitis virus infection in Dicer1-deficient mice [39]. miR-93 promotes the axon growth of spinal cord neurons by targeting to EphA4 [40]. In proliferation, miR-93 promotes the proliferation of osteosarcoma cells by targeting PTEN [41, 42].

Nevertheless, how microRNA-modulated key autophagic machinery is involved in hypoxic condition remains poorly understood. Here, we uncovered the regulating mechanism between miRNA-93, ULK1, and hypoxiainduced autophagy.

\section{Materials and Methods}

2.1. Reagents and Antibodies. DMEM (Gibco, C11965500BT), fetal bovine serum (Gibco, 16000044), EBSS (MACGENE, CC026), FITC Annexin V apoptosis detection kit I (BD Biosciences, 556547), cell counting kit (YEASEN, QF0026), lysis buffer (Beyotime, P0013), PVDF membranes (Millipore, ISEQ00010), albumin bovine V (Solarbio,
A8020), lipofectamine 2000 (Invitrogen, 11668027), DualLuciferase $^{\circledast}$ Reporter Assay System (Promega, E1910), and terminal deoxynucleotidyl transferase-mediated dUTP nick-end labeling (TUNEL) staining kit (Promega, G3250). Bafilomycin A1 was purchased from Sigma. The following antibodies were used: anti-ULK1 (Sigma-Aldrich, A7481), anti-P62 (MBL, PM045), anti-P62 (Abcam, ab56416), anti-LC3B (Sigma, L7543), anti-c-Myc monoclonal antibody (Sigma, C3956), anti- $\beta$-actin (Sino Biological, 100162-RP02100), anti-Gapdh (Transgen, HC301), anti-ULK1 (Sigma, A7481), anti-ATG5 (Sigma, A0731), anti-ATG7 (Sigma, A2856), anti-Beclin1 (CST, 3738), anti-Tom20 (BD Biosciences, 612278), DAPI (CST, 4083), HRP Affinipure goat anti-mouse IgG (Earthox, E030110), and HRP Affinipure goat anti-rabbit IgG (Earthox, E030120). The following fluorescent secondary antibodies were used: Alexa Fluor 555-labeled donkey anti-mouse IgG antibodies (Invitrogen, A31570) and Alexa Fluor 488-labeled donkey anti-rabbit IgG antibodies (Invitrogen, A21206).

2.2. Cell Culture, Hypoxia, Starvation Treatment, and Transfection. The methods we used in the Materials and Methods were followed from our previous paper by Li et al. [43]. MEFs (mouse embryonic fibroblasts), CHO (Chinese hamster ovary cell), and HeLa cells were used in this study. Cells were cultured in Dulbecco's modified Eagle's medium supplemented with $10 \%$ FBS and 1\% penicillin/ streptomycin (Beyotime, referred to as complete medium) at $37^{\circ} \mathrm{C}$ and $5 \% \mathrm{CO}_{2}$. To determine the levels of autophagy, we starved cells for different times with EBSS. Hypoxic conditions were achieved with a hypoxia chamber (BillupsRothenberg) flushed with a preanalyzed gas mixture of $1 \%$ $\mathrm{O}_{2}, 5 \% \mathrm{CO}_{2}$, and $94 \% \mathrm{~N}_{2}$. Cell transfection was performed using lipofectamine 2000 according to protocols provided by manufacturers.

2.3. Plasmids. Myc-ULK1 constructs were described previously. The $3^{\prime}$ untranslated region (UTR) of ULK1 (NM_009469) was amplified from mouse cDNA. The primers used were as follows: $5^{\prime}$-GCGCTAGCCCAGGG GTCCCTTGCCCAC-3' (UTR, F), 5'-GGTCTAGAGTAAA GTGTGGAAGTTGAGG-3' (UTR, R). PCR products were cloned into pmirGLO dual-luciferase miRNA target expression vector (Promega) using NheI and $\mathrm{XbaI}$ restriction sites, named pmirGLO-ULK1. The corresponding binding site mutation was also cloned using the following primers: $5^{\prime}-$ TTTGTAAGTCACCGGTAACTGCCATGCATACAGAGA CTGGA-3' (M-UTR, F), 5'-TCCAGTCTCTGTATGCATG GCAGTTACCGGTGACTTACAAA-3' (M-UTR, R). Mutation, named mutant pmirGLO-ULK1, was created by sitedirected mutagenesis, changing "gcacttt” to “aactgcc.” Q5 ${ }^{\circledR}$ High-Fidelity DNA Polymerase (Bio Labs, M0491S) was used for PCR reaction. The plasmids were sequenced to verify the accuracy.

2.4. siRNAs and miRNAs. Five pairs of siRNAs were designed and synthesized as follows by GenePharma: siULK1-Mus-488 (sense: CCGUCAAAUGCAUUAACAATT, antisense: UUGUUAAUGCAUUUGACGGTT), si-ULK1- 
Mus-2457 (sense: GCUGCUUAAGGCUGCAUUUTT, antisense: AAAUGCAGCCUUAAGCAGCTT), si-ULK1-Mus3310 (sense: GCUGUGCAAAUGGUACAAUTT, antisense: AUUGUACCAUUUGCACAGCTT), and negative control (NC) and negative control FAM (sense: UUCUCCGAACG UGUCACGUTT, antisense: ACGUGACACGUUCGGAGA ATT). miR-93 mimics (93) and miR-93 inhibitor (IN-93) were synthesized by RiboBio (Guangzhou, China) with reference to miRNA's sequence information in miRbase. Meanwhile, NControl (NC) and inhibitor NControl (IN-NC) were also synthesized as a negative control.

2.5. Luciferase Assay. Cells were seeded in 24-well plates 1 day before transfection. For reporter assays, the cells were transiently cotransfected with $0.8 \mu \mathrm{g}$ of reporter plasmid in the presence of $100 \mathrm{nM}$ scramble NC (NC), miR-93 mimics (93), NC inhibitor (IN-NC), or miR-93 inhibitor (IN-93) using lipofectamine 2000. Firefly and Renilla luciferase activities were measured consecutively by using dual-luciferase reporter assay system according to the manufacturer's instructions. Independent experiments were performed in triplicate.

2.6. $q R T-P C R$. Total RNA was isolated with TRIzol (Life Technologies) followed by a DNase treatment to eliminate contaminating genomic DNA (Thermo, B43) and reverse transcription reaction (Thermo, K1622). Amplification and relative quantification of cDNA was carried out with SYBR ${ }^{\oplus}$ Premix Ex Taq ${ }^{\mathrm{TM}}$ (Tli RNaseH Plus) (TaKaRa, RR420A) according to the manufacturer's protocol. Relative quantitative PCRs for miRNAs were performed with SYBR PrimeScript miRNA RT-PCR Kit (TaKaRa, RR716) as described before. Fold changes were calculated using the $2^{-\Delta \Delta \mathrm{Ct}}$ method with normalization to endogenous control. Primers used were as follows: 5'-CTCGCTTCGGCAGCACA-3' (U6F), 5'-AACGCTTCACGAATTTGCGT-3' (U6-R). miR-93 qPCR primers were purchased from RiboBio (Guangzhou, China). 5'-CCAGGCAGACATTGAGAACA-3' (ULK1-F), 5'-GTTGGCAGCAGGTAGTCAGG-3' (ULK1-R), 5'-CCAC CCAGAAGACTGTGGAT- ${ }^{\prime}$ (Gapdh-F), 5'-CACATTGGG GGTAGGAACAC-3' (Gapdh-R). Independent experiments were performed in triplicate.

2.7. Western Blotting. Whole cell lysates used for immunoblotting were prepared in lysis buffer containing a phosphatase inhibitor (Roche, 4693116001) on ice. Lysates were mixed with SDS loading buffer and boiled for $10 \mathrm{~min}$. Protein samples were separated on SDS-PAGE gels and then transferred to PVDF membrane. Membranes were blocked by $5 \%$ nonfat milk (dissolved in PBST) for $1 \mathrm{~h}$ at room temperature. Corresponding primary antibody was used for overnight incubation at $4^{\circ} \mathrm{C}$, followed by HRPlabeled secondary antibodies' incubation at room temperature for $2 \mathrm{hrs}$. For loading controls, membranes were probed with the antibody against $\beta$-actin or Gapdh. Densitometric ratios were quantified by ImageJ software.

2.8. Immunofluorescence Microscopy. Cells were grown to $60 \%$ confluence on a coverslip. After treatment, the cells were washed in PBS and fixed with $4 \%$ paraformaldehyde for
20 mins. After washed twice in PBS, the cells were permeabilized with $0.1 \%$ Triton X-100 (in PBS). Coverslips were blocked in 1\% albumin bovine V (in PBS) for 30 mins at room temperature and then were incubated with primary antibody diluted in PBS (0.01\% Triton X-100) for $1 \mathrm{~h}$ at room temperature. After washed with PBS for $4 \times 5$ mins, secondary antibodies were applied. Cell images were captured with a TCS SPF5 II Leica confocal microscope and software (LAS-AF-Lite_2.2.0_4758, Germany).

2.9. CCK-8 Assay. Cell viability was measured using the CCK-8 kit. Briefly, $7 \times 10^{6}$ cells in the logarithmic growth phase were seeded into a 24 -well plate and incubated in $500 \mu \mathrm{L}$ complete medium overnight. MEFs and HeLa cells were transfected with scramble NC (NC), miR-93 mimics (93), NC inhibitor (IN-NC), or miR-93 inhibitor (IN-93) individually for $12 \mathrm{~h}$, followed by the treatment of hypoxia for another $24 \mathrm{~h}$. Moreover, MEFs were transfected with scramble NC (NC), miR-93 mimics (93), NC inhibitor (IN-NC), or miR-93 inhibitor (IN-93) individually for $24 \mathrm{~h}$, followed by subjecting to starvation for another $1 \mathrm{~h}$. The cell viability was detected according to the manufacturer's instructions. The optical density (OD) at $450 \mathrm{~nm}$ was measured using a microplate spectrophotometer (Biotek, Epoch). Cell viability was presented using the normal group as control.

2.10. Flow Cytometry. Cell apoptosis was also assessed by flow cytometry after Annexin V-FITC/PI staining as manufacturer's instructions. MEFs were transfected with scramble NC (NC), miR-93 mimics (93), NC inhibitor (IN-NC), or miR-93 inhibitor (IN-93) individually for $12 \mathrm{~h}$, followed by the treatment of hypoxia for another $24 \mathrm{~h}$. The normal group was set as control. Analyses were carried out with a COULTER EPICS XL-MCL ${ }^{\mathrm{TM}}$ flow cytometer (Beckman Coulter) equipped with Expo32 ADC analysis software. The cellular apoptosis rate was mapped by GraphPad Prism 5.

2.11. TUNEL Assay. Briefly, HeLa cells were transfected with scramble NC (NC), miR-93 mimics (93), NC inhibitor (IN-NC), or miR-93 inhibitor (IN-93) individually for $12 \mathrm{~h}$, followed by the treatment of hypoxia for another $24 \mathrm{~h}$. Analysis of apoptotic cells was performed using the TUNEL staining kit following the manufacturer's instruction, using normal group as control. Cell images were captured with an EVOS FL Auto Cell Imaging System (Thermo Fisher Scientific). The nuclei were counterstained with 4',6-diamidino-2-phenylindole (DAPI) (blue). TUNEL-positive cells had a pyknotic nucleus with dark green fluorescent staining, indicative of apoptosis.

\section{Results}

3.1. miR-93 Targets ULK1 and Regulates Its Expression. ULK1 is the initiation factor of autophagy, which belongs to the family of serine/threonine kinases and is essential for the induction of autophagy $[44,45]$. However, ULK1modulating microRNAs are not well understood. In order to determine the miRNA that targets ULK1, we used DIANALAB to perform bioinformatics search. Interestingly, the 
software showed that ULK1 is the target of miR-93. Prediction of the interaction between miR-93 and $3^{\prime}$ UTR of ULK1 was shown in Figure 1(a). ULK1 contains a miR-93 binding site in the $3^{\prime}$ UTR (302-309 nucleotides). We then used the dual luciferase carrier pmirGLO to verify whether the miR-93 can identify and bind the $3^{\prime}$ UTR of ULK1. The $3^{\prime}$ UTR of ULK1 was cloned and inserted into the downstream of the firefly luciferase of plasmid pmirGLO. $100 \mathrm{nM}$ scramble NC (NC), miR-93 mimics (93), NC inhibitor (IN-NC), or miR-93 inhibitor (IN-93) and a reporter gene were cotransfected into MEFs, respectively. As shown in Figure 1(b), the expression of miR-93 inhibited the firefly luciferase enzyme activity. However, the IN-93 could restore the activity of luciferase, suggesting the strong inhibitory effect of endogenous miR-93. Real-time quantitative PCR (qPCR) was performed by using U6 and Gapdh as the benchmark to detect the expression of miR-93 and ULK1 in either MEFs or CHO cells. The results showed that overexpression of exogenous miR-93 mimics or miR-93 inhibitor group was successful. ULK1 and miR-93 were individually inhibited by 93 and IN-93 (Figures 1(c) and 1(d)). The NC, 93, IN-NC, and IN-93 were also transfected into the cells for $24 \mathrm{~h}$ to detect the protein expression of ULK1 to confirm the inhibitory effect of miR-93 by Western blotting. miR-93, respectively, decreased the expression of ULK1 by $32 \%$ (in MEFs) and 70\% (in CHO cells). Nevertheless, miR-93 inhibitor increased the expression of ULK1 by $28 \%$ (in MEFs) and 32\% (in CHO cells) (Figures 1(e) and 1(f)). In order to exclude the off-target effects of miR-93 and to monitor a time course of the inhibitory effect of miR-93 to ULK1, some other autophagy-related genes were also examined by Western blotting. The expression of ATG5, ATG7, Beclin1, or Tom20 did not change when exogenously overexpressed with miR-93 and did not increase while exogenously overexpressed with miR-93 inhibitor (IN-93) (Figures $1(\mathrm{~g})$ and $1(\mathrm{~h})$ ).

3.2. miR-93 Suppresses Hypoxia-Induced Autophagy. In order to find out whether miR-93 regulates the expression of ULK1 under the condition of hypoxia, cells were divided into four groups according to the time of hypoxia. Similarly as before, the protein levels of ULK1, P62, and LC3 transition confirmed that hypoxia could induce autophagy (Figure 2(a)). In hypoxia groups, the expression levels of ULK1 and LC3I to LC3-II transition were increased but the expression of P62 was reduced (Figure 2(a)). The transcriptional and translational levels of ULK1 were higher than those in the normal group (Figures 2(a) and 2(b)). In contrast, the expression of miR-93 decreased during hypoxia (Figure 2(b)). Based on the above findings, we hypothesized that miR-93 might play an important role in hypoxia-induced autophagy. We then transfected scramble NC (NC), miR-93 mimics (93), NC inhibitor (IN-NC), or miR-93 inhibitor (IN-93) to examine their roles in hypoxia-induced autophagy. We found that miR-93 caused the downregulation of ULK1 as well as upregulation of P62, and decreased the ratio of LC3-II to LC3-I under hypoxic condition (Figure 2(c)). In contrast, this effect could be reversed by the endogenously overexpressed IN-93 during hypoxia. The expression of ULK1 in miR-93 group was lower than that in NC group. Compared to IN-NC group, IN-93 group promoted the expressional level of ULK1 (Figure 2(c)). Lysosomal inhibitor Bafilomycin A1 (BAF1) prevented the transition of LC3 and $\mathrm{P} 62$ protein degradation under hypoxic condition, indicating that hypoxia promotes the improvement of autophagic flux. The densitometric ratios from the samples were quantified by using ImageJ (Figure 2(c)). The results were further examined by immunofluorescence. The samples were stained with antiULK1 (green) and anti-P62 (red) antibodies. miR-93 inhibited the expression of ULK1 as well as its puncta formation during hypoxia (Figure 2(d)). On the contrary, miR-93 increased the P62 puncta under the same condition. Conversely, this effect could be reversed by IN-93, an inhibitor of endogenous miR-93. Upon inhibition of endogenous miR-93, ULK1 puncta increased accompanied by decreased expressional level of P62 (Figure 2(d)).

3.3. The Knockdown of ULK1 by siRNA Suppressed HypoxiaInduced Autophagy. From the above results, we knew that hypoxia could trigger the decrease of miR-93 and the increase of ULK1 expression and consequently induce autophagy. Therefore, the modulatory effect of miR-93 on ULK1 might become less relevant since the weakened expression of miR-93 under hypoxic condition. To determine the relevance of miR-93 in the regulation of ULK1, siRNA of ULK1 was used to reveal that if knocking down of ULK1 had the same effect similar to miR-93 in hypoxia-induced autophagy. It is clear that siRNA, a class of double-stranded RNA molecules, interferes with the expression of specific genes by degrading mRNA after transcription (via complementary nucleotide sequences), resulting in no translation $[46,47]$. Four pairs of siRNAs (including negative control) were designed, synthesized, and transfected into MEFs and $\mathrm{CHO}$ cells. Fortunately, we found the most effective siRNA interference site (488) attributing to its interference efficiency in MEF cells $(\sim 40 \%)$ and $\mathrm{CHO}$ cells $(\sim 60 \%)$ (Figures $3(\mathrm{a})$ and 3(b)). We then transfected si-ULK1 (488) and negative control RNA (NC) to examine their roles in hypoxia-induced autophagy. We found that si-ULK1 caused the downregulation of ULK1 as well as upregulation of P62 and decreased the ratio of LC3-II/LC3-I under hypoxic condition. The expression of ULK1 in si-ULK1 group was lower than that in NC group. BAF1 prevented the transition of LC3 and P62 protein degradation under hypoxic condition. The densitometric ratios from the samples were quantified by using Image (Figure 3(c)). The results were further examined by immunofluorescence. The samples were stained with antiULK1 (green) and anti-P62 (red) antibodies. si-ULK1 inhibited the expression of ULK1 as well as its puncta formation during hypoxia. On the contrary, si-ULK1 increased the P62 puncta under the same condition (Figure 3(d)).

3.4. Re-Expression of ULK1 without miR-93 Binding Elements Restores Autophagy Inhibited by miR-93. In order to verify that the inhibition of ULK1 is due to miR-93, we evaluated the expression of ULK1-Myc (CDS) which lacked the miR-93 recognition element so that it can resist miR-93-mediated inhibition. ULK1-Myc (CDS) transfection 


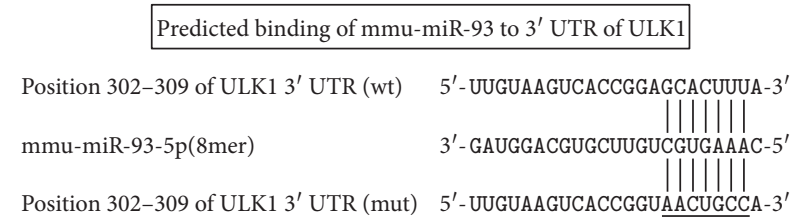

(a)

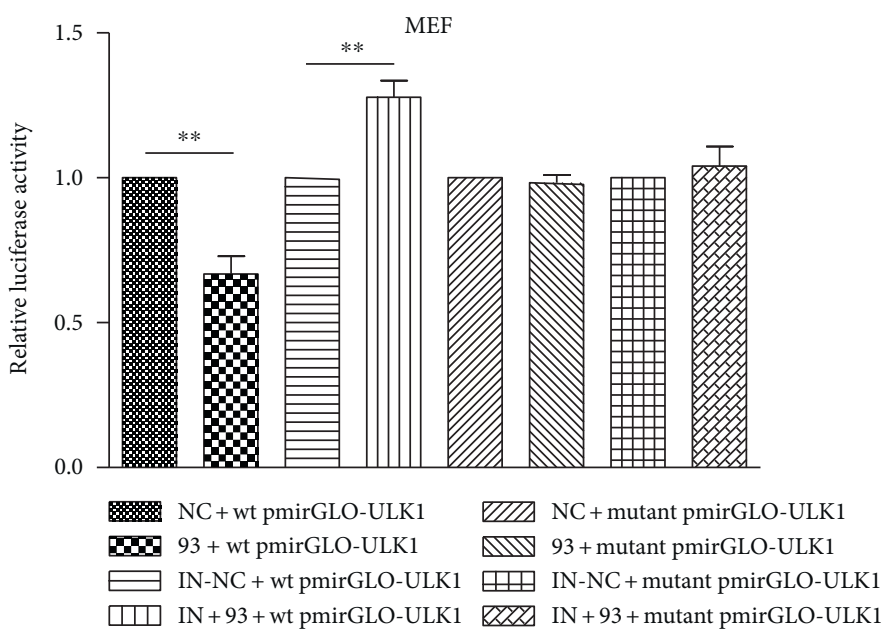

(b)
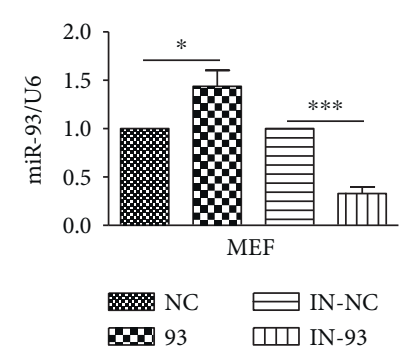

(c)

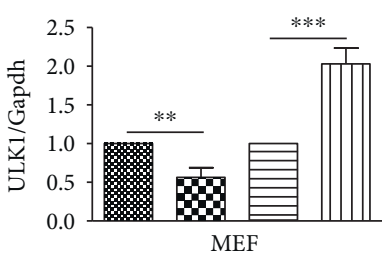

\%थ NC $\rightleftharpoons$ IN-NC 표미 IN-93
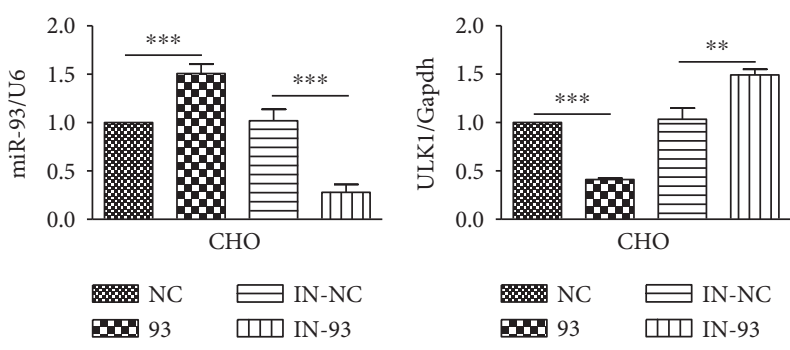

(d)

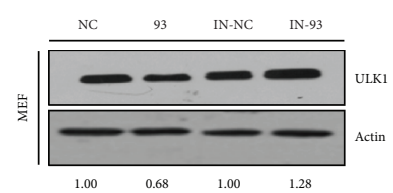

(e)

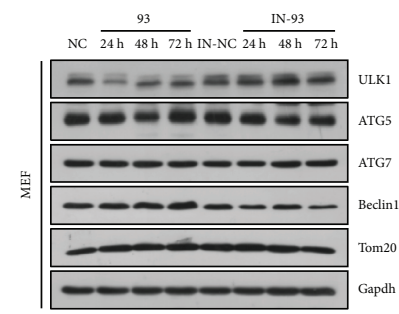

(g)

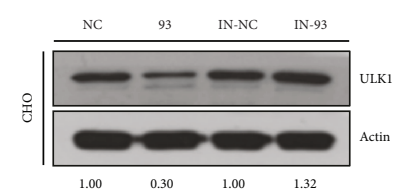

(f)

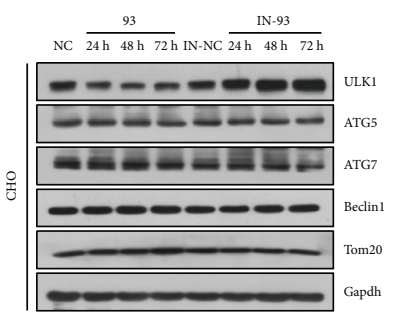

(h)

FIGURE 1: miR-93 targets ULK1 and regulates its expression. (a) Predicted binding sites of mmu-miR-93 to the $3^{\prime}$ UTR of ULK1. The short vertical lines indicate complementary paired bases of miR-93 and ULK1. The 302-309 nucleotides of ULK1 $3^{\prime}$ UTR present the miRNA regulatory element (MRE). The underlined bases were mutant MRE. (b) Luciferase reporter assay by the interaction between miR-93 and the predicted MRE in MEFs. Each luciferase construct was cotransfected with scramble NC (NC), scramble IN-NC (IN-NC), miR-93 mimics (93), or miR-93 inhibitor (IN-93). At approximately $24 \mathrm{~h}$ after transfection, the luciferase activity was detected. The firefly luciferase activity was normalized to Renilla. Data were shown as the mean \pm S.D. from three independent experiments. ${ }^{* *} P<0.01$. (c) miR-93 represses the endogenous ULK1 expression in MEFs. NC, 93, IN-NC, and IN-93 were transfected into MEFs. At $24 \mathrm{~h}$ after transfection, qPCR was performed to detect the expression of miR-93 and ULK1. Data were from three independent experiments. ${ }^{*} P<0.05 ;{ }^{* *} P<0.01 ;{ }^{* * *} P<0.001$. (d) miR-93 represses the endogenous ULK1 expression in CHO cells. NC, 93, IN-NC, and IN-93 were transfected into $\mathrm{CHO}$ cells. At $24 \mathrm{~h}$ after transfection, qPCR was performed to detect the expression of miR-93 and ULK1. Data were from three independent experiments. ${ }^{* *} P<0.01$; ${ }^{* * *} P<0.001$. (e) Cell lysates in (c) were prepared and subjected to Western blot analysis by using anti-ULK1 and anti- $\beta$-actin antibody. The densitometric ratios of ULK1/actin from the samples were quantified by using ImageJ. Data were from three independent experiments. Representative data are shown. (f) Cell lysates in (d) were prepared and subjected to Western blot analysis by using anti-ULK1 and anti- $\beta$-actin antibody. The densitometric ratios of ULK1/actin from the samples were quantified by using ImageJ. Data were from three independent experiments. Representative data are shown. (g, h) MEFs and CHO cells were transfected with miR-93 (93) and miR-93 inhibitor (IN-93) for $24 \mathrm{~h}, 48 \mathrm{~h}$, and $72 \mathrm{~h}$, individually, setting group NC as a control. Cell lysates were prepared and subjected to Western blot analysis by using anti-ULK1, anti-ATG5, anti-ATG7, anti-Beclin1, anti-Tom20, and anti-Gapdh. Data were from three independent experiments. Representative data are shown. 


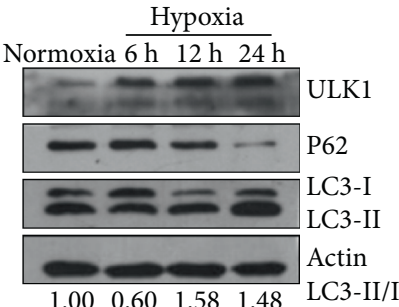

(a)
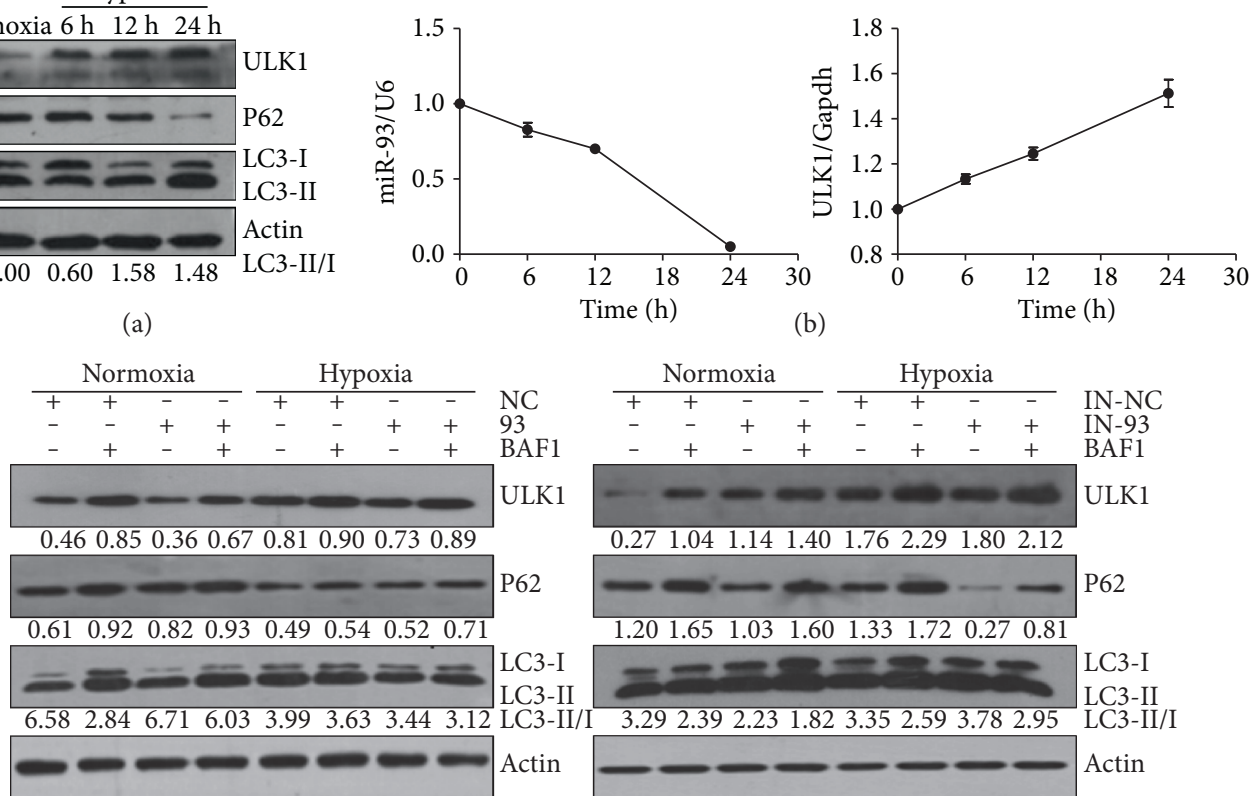

(c)

miR-93 reduces hypoxia induced autophagy via targeting ULK1 BAF1

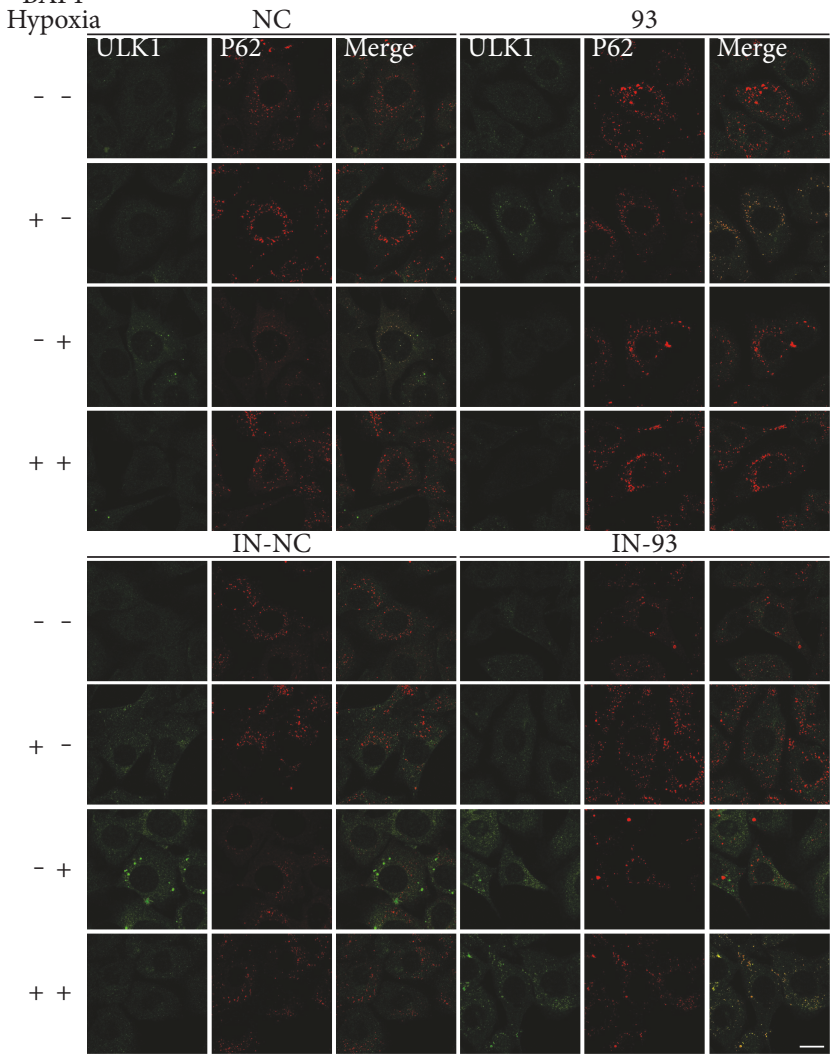

(d)

FIGURE 2: miR-93 inhibits hypoxia-induced autophagy. (a) The expression of ULK1 is induced by hypoxia. MEFs are subjected to hypoxia conditions for $0 \mathrm{~h}, 6 \mathrm{~h}, 12 \mathrm{~h}$, and $24 \mathrm{~h}$. Endogenous ULK1, P62, LC3, and $\beta$-actin were blotted by corresponding antibodies. Densitometric ratios of LC3-II/I from the samples were quantified by using ImageJ. (b) Expression levels of endogenous miR-93 and ULK1 in MEFs under hypoxia condition for $0 \mathrm{~h}, 6 \mathrm{~h}, 12 \mathrm{~h}$, or $24 \mathrm{~h}$ by qPCR. (c) MEFs were transfected with scramble NC (NC), miR-93 mimics (93), scramble IN-NC (IN-NC), or miR-93 inhibitor (IN-93) for $12 \mathrm{~h}$; afterward, MEFs were sealed in a hypoxic or normoxic condition for another $24 \mathrm{~h}$ with or without Bafilomycin A1 (BAF1). Samples were blotted with anti-ULK1, anti-P62, anti-LC3, and anti- $\beta$-actin antibodies. (d) MEFs were treated as the same as (c). Cells were fixed and immunostained with anti-ULK1 (green) and anti-P62 (red) antibodies. Scale bar, $20 \mu \mathrm{m}$. 


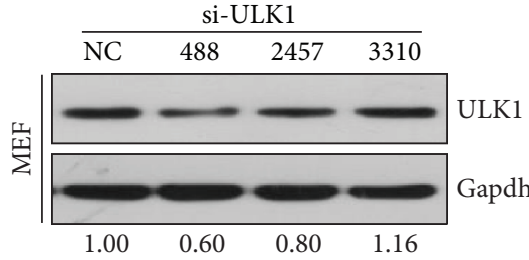

(a)

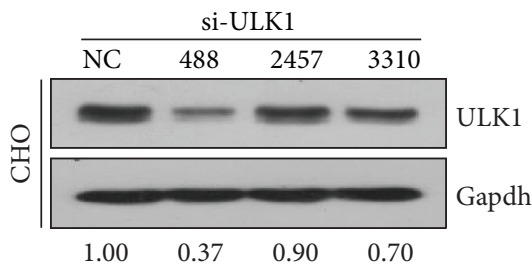

(b)

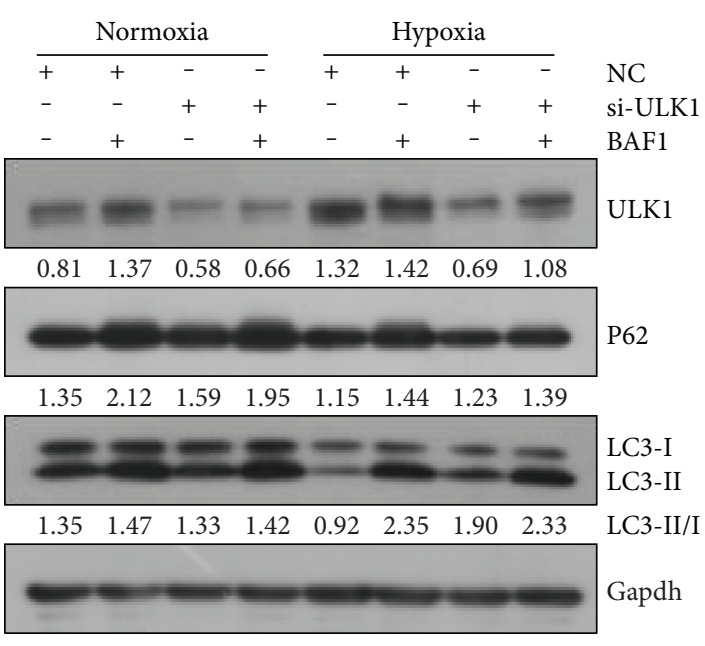

(c)
BAF1

Hypoxia

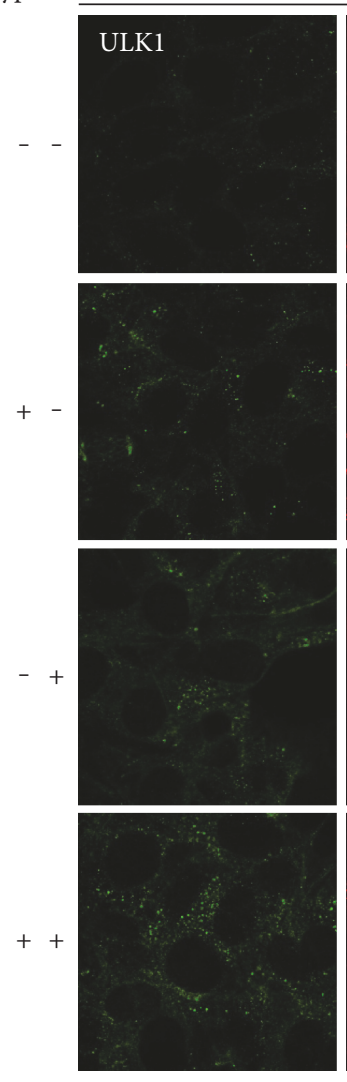

NC
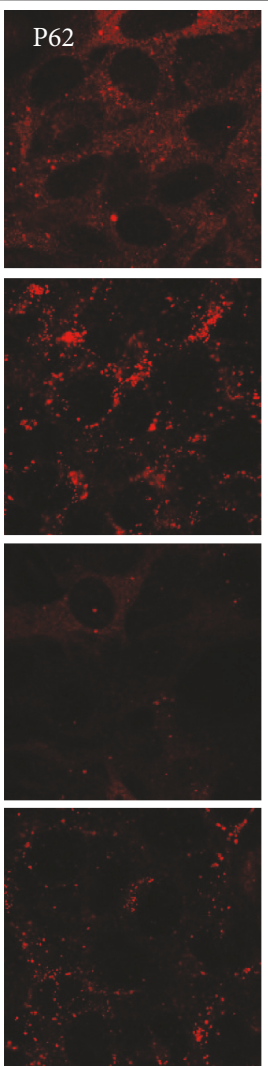

si-ULK1
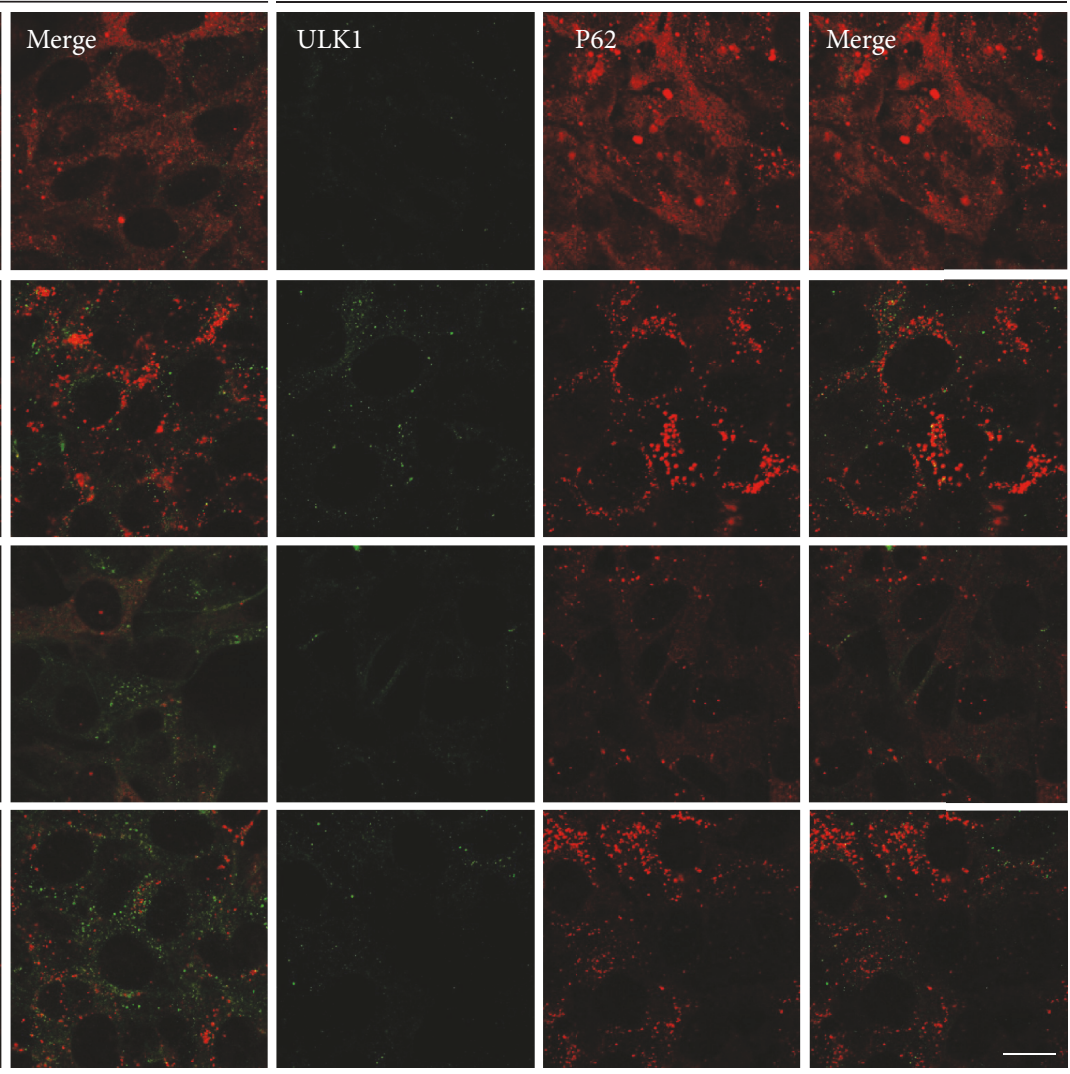

(d)

FIGURE 3: The knockdown of ULK1 by siRNA suppressed hypoxia-induced autophagy. (a, b) MEFs and CHO cells were transfected with three pairs of siRNAs to ULK for $24 \mathrm{~h}$, respectively, si-ULK1 (488), si-ULK1 (2457), and si-ULK1 (3310), setting NC as a control. Cell lysates were prepared and subjected to Western blot analysis by using anti-ULK1 and anti-Gapdh. The densitometric ratios from the samples were quantified by using ImageJ. Data were from three independent experiments. Representative data are shown. (c) Si-ULK1 (488) was transfected into MEFs for $12 \mathrm{~h}$, followed by hypoxic treatment or normoxic treatment for another $24 \mathrm{~h}$. Cell lysates were analyzed by Western blot using anti-ULK1, anti-P62, anti-LC3, and anti-Gapdh. Densitometric ratios of the samples were quantified by using ImageJ. (d) MEFs were treated as the same as (c). Cells were fixed and immunostained by anti-ULK1 (green) and anti-P62 (red) antibodies. Scale bar, $20 \mu \mathrm{m}$. 
effectively induced the P62 degradation and LC3-I to II transition even in the presence of miR-93 under hypoxia (Figure 4(a)). In order to distinguish the immunoreactivityderived signal from the background staining, MEFs were fixed, permeabilized, and only stained with the second antibody donkey anti-rabbit 488 or donkey anti-mouse 555 . As seen from Figure 4(b), there was no fluorescence signal of MEFs. Consistent with Figure 4(a), immunofluorescence results revealed that ULK1-Myc could effectively induce P62 degradation which was inhibited by exogenous overexpression of miR-93 under hypoxia as confirmed by WB (Figure 4(b)).

3.5. The Effects of miR-93 on Cell Viability and Apoptosis in Noncancer Cell Lines and Cancer Cells. Some genes involved in cell death are associated with the autophagy pathway. Excessive autophagy leads to cell death due to excessive digestion of essential intracellular proteins and organelles, which triggers the death signal [48-50]. Considering that miR-93 is involved in hypoxia by targeting key autophagy proteins, we assessed whether miR-93 is involved in cell viability in nutrient-depleted condition or during hypoxia. We used the cell counting kit to analyze and evaluate the cell viability during either condition. The cell viability of MEFs decreased to about $65 \%$, in these conditions (Figures 5(a) and 5(b)). Upon starvation, the cell viability of MEFs did not change notably after the exogenous overexpression of miR-93 or miR-93 inhibitor, compared to $\mathrm{NC}$ or IN-NC group. Although the cell viability of MEFs between NC + starvation group and IN-NC + starvation group seemed to be different, there was no significant difference between the two groups $(P=0.09)$ (Figure 5(a)). Unlike starvation condition, in hypoxic condition, the miR-93 group recovered the cell viability from $65 \%$ to $136 \%( \pm 6 \%)$ compared with $\mathrm{NC}$ group, while IN-93 further dampened the cell viability of MEFs to about $74 \%( \pm 21 \%)$ during hypoxia, (Figure 5(b)). Although the cell viability of MEFs between $\mathrm{NC}+$ hypoxia group and IN-NC + hypoxia group seemed to vary, there was no significant difference between the two groups $(P=0.21)$ (Figure 5(b)). We subsequently examined whether miR-93 protected the cells from hypoxic stress by weakening cell apoptosis. Flow cytometry analysis revealed that miR-93 decreased hypoxia-induced cell apoptosis of MEFs, which was reversed by miR-93 inhibitor (IN-93) (Figure 5(c)). Given that autophagy and hypoxia are implicated to play an important role in cancer, we further investigated miR93-based regulation of ULK1, and hypoxia-mediated modulation of miR-93 in human cancer cells. HeLa cells were used to take cell viability test and TUNEL assay. As shown in Figure 5(d), the cell viability of HeLa was dramatically reduced under hypoxic condition, compared with normal group. Interestingly, the exogenous overexpressed miR-93 (93) further accelerated the decrease of cell viability under the stress of hypoxia. By contrast, miR-93 inhibitor (IN-93) could reverse the cell viability by reducing the expression of endogenous miR-93. In view of the interesting experimental phenomena mentioned above, we did the TUNEL assay afterwards. Nevertheless, the overall apoptosis differences between groups were not significant (Figure 5(e)).

\section{Discussion}

Here, we found that the expression of ULK1 was upregulated but miR-93 level was declined under hypoxic condition. Their expression patterns showed a negative correlation (Figure 2). By using LAB DIANA, we uncovered a direct relationship of miR-93 and ULK1. The $3^{\prime}$ UTR of ULK1 had miR-93 pairing sites. The luciferase activity assay, quantitative RT-PCR, and Western blotting results showed that miR-93, when overexpressed, regulated ULK1 at the transcriptional and posttranscriptional levels. The inhibitory effect of miR-93 on ULK1 was also observed under hypoxic condition (Figure 2). Immunofluorescence showed that the expression of ULK1 was declined and the P62 level was increased by miR-93 (Figure 2). To determine the relevance of miR-93 in the regulation of ULK1, siRNA of ULK1 was used to reveal that knockdown of ULK1 had the same effect as compared to miR-93 in hypoxia-induced autophagy. Data showed that hypoxia induced autophagy was indeed suppressed after interference with ULK1 (Figure 3). This is consistent with the results from miR-93. In order to verify that the inhibition of ULK1 was caused by miR-93, we assessed whether the ULK1 plasmid lacking the miR-93 recognition element was able to resist miR-93-mediated inhibition. Immunofluorescence and Western blotting indicate that ULK1 plasmid that lacks miR-93 recognition element can rescue the autophagy inhibited by miR-93 (Figure 4).

Substantial progress helps us to understand the molecular mechanism of autophagy but there are still a lot of unknowns needed to be further clarified. Recent evidence that most autophagy genes can be regulated by miRNAs increases a more complicated cellular layer control of autophagy. Autophagy and microRNAs seem to have mutual communications with each other. On one hand, microRNAs regulate autophagy by directly targeting the autophagy genes including Beclin1 [51, 52], ATG3 [53], or ATG5 [54] as well as mitophagy genes, FUNDC1 and NIX [29]. On the other hand, autophagy activity modulates miRNA-mediated gene silencing and degrades the core miRISC component [55].

We found the negative correlation of miR-93 and ULK1. While ULK1 is induced and miR-93 is decreased under hypoxia. Interestingly, Hazarika et al. reported that hypoxia induced an increased expression of miR-93 [56], but Ke and colleagues observed a decreased level of miR-93 [41], which is consistent with our observations. We thought that the discrepancy may be due to the use of different cell types.

The cellular level of ULK1 is coordinately controlled by a variety of mechanisms. During early stages of autophagy, the E3 ligase complex AMBRA1-TRAF6 mediates K63-linked polyubiquitination of ULK1 to maintain its stability and kinase activity [57]. On the contrary, Cullin/KLHL20 catalyzes K48-linked polyubiquitination of ULK1 for proteasome degradation during prolonged nutrient starvation, thus providing negative feedback loop of the autophagy control [17]. Recently, Nazio et al. provide evidence that levels and activity of ULK1 are temporally controlled by NEDD4Lmediated degradation and mTOR-dependent de novo protein synthesis to modulate the duration of the autophagy response during prolonged starvation [19]. Similarly, the 


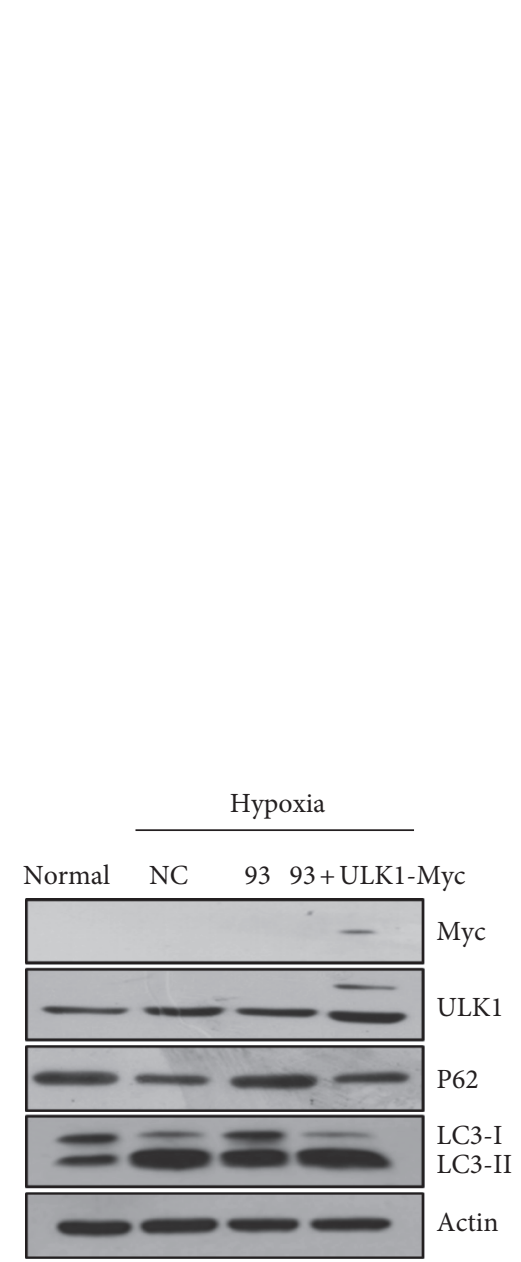

(a)
Normoxia
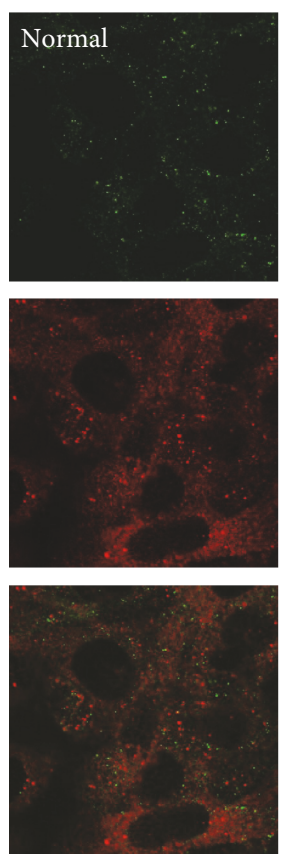

Control

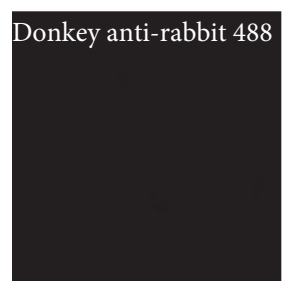

Hypoxia
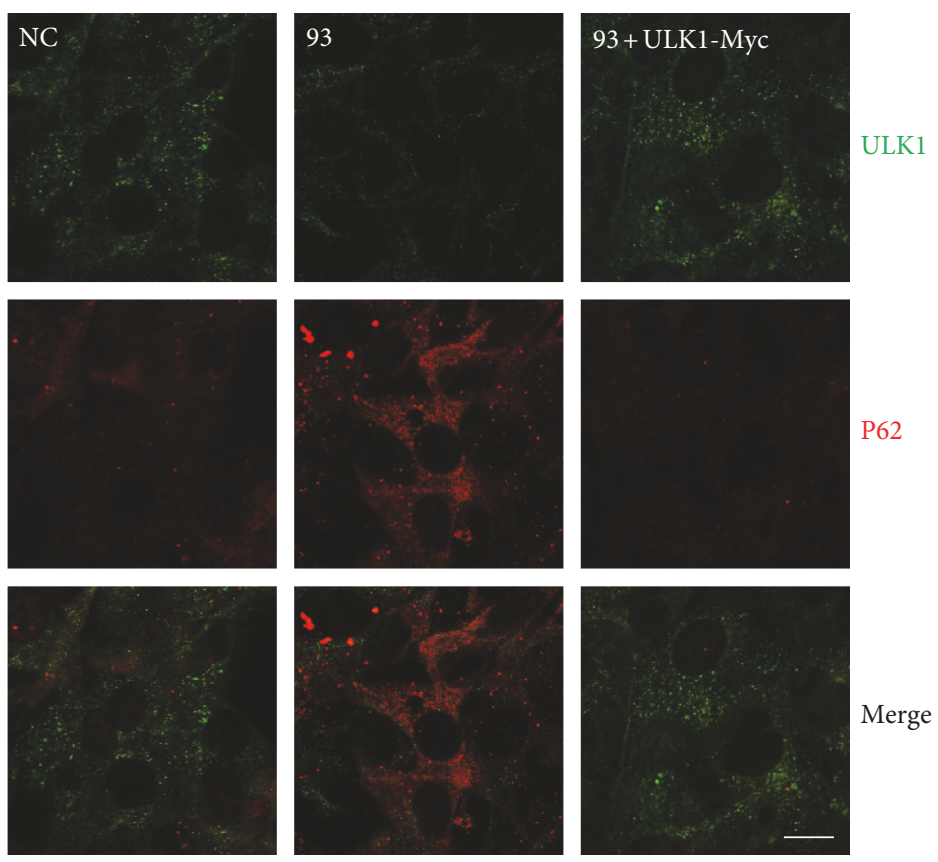

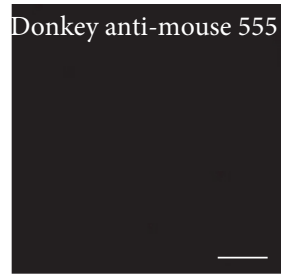

(b)

FIGURE 4: Re-expression of ULK1 without miR-93 response elements restores autophagy inhibited by miR-93. (a) MEFs were transfected with scramble NC (NC) and miR-93 mimics (93) or cotransfected miR-93 mimics (93) with the indicated constructs (ULK1-Myc) for 24 h by using the normal group as a control. Protein samples were collected and the expression of ULK1, Myc, LC3, and P62 was analyzed through Western blot. Actin was used as a loading control. (b) MEFs were fixed and immunostained by donkey anti-rabbit 488 or donkey anti-mouse 555 . Scale bar, $20 \mu \mathrm{m}$. (c) MEFs were treated the same as (a). Cells were fixed and immunostained by anti-ULK1 (green) and anti-P62 (red) antibodies. Scale bar, $20 \mu \mathrm{m}$.

ULK1 expression is induced under hypoxia treatment. Our previous results demonstrate that the level of ULK1 is regulated by hypoxia from tens of minutes to 24 hours $[15,16]$. But the expression of ULK1 declines if the hypoxic time is longer than 36 hours, indicating that the ULK1 expression is controlled by multiple cellular factors.

In this study, we have discovered that ULK1 is a new target gene of miR-93. Unlike the effect of miR-93 in nutrient-deprivation condition, our results show that miR93 was able to recover the cell viability and lower the rate of apoptosis compared to the NC group under hypoxic condition in noncancerous MEFs, indicating that miR-93 might contribute some beneficial effects to this kind of cells under hypoxia. However, the effect of miR-93 in cancerous cell line HeLa is quite different. The cell viability of HeLa was dramatically reduced under hypoxic condition, compared with normal group. But, the exogenous overexpressed miR-93 (93) further accelerated the decrease of cell viability under the stress of hypoxia. By contrast, miR-93 inhibitor (IN-93) could revert the cell viability by reducing the expression of endogenous miR-93. The data suggests that autophagy might be more important for sustaining the viability of the cancerous cell line than noncancer cell lines during stressful hypoxia condition. Nevertheless, the overall apoptosis differences between groups were not significant (Figure 5). The different cell viability results of miR-93 in cancerous and noncancerous cell lines under hypoxia condition demonstrated a quite complex regulation of microRNA, autophagy, and cells that undergo hypoxic stress. This may be because of that the metabolism and oxygen consumption rate varies in cancerous and noncancerous cell lines. The detailed molecular mechanism needs to be further investigated.

We also observed that hypoxia substantially increases autophagy flux by upregulating the levels of ULK1 and suppresses the expression of miR-93 (Figure 2). This study provides a novel insight into how the oscillation level of 


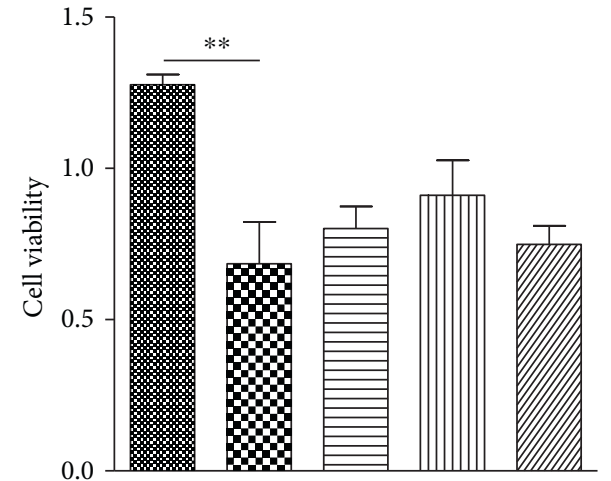

\% Normal $\square$ IN-NC + starvation D. NC + starvation $\mathbb{Z I Z I}$ IN-93 + starvation 93 + starvation

(a)
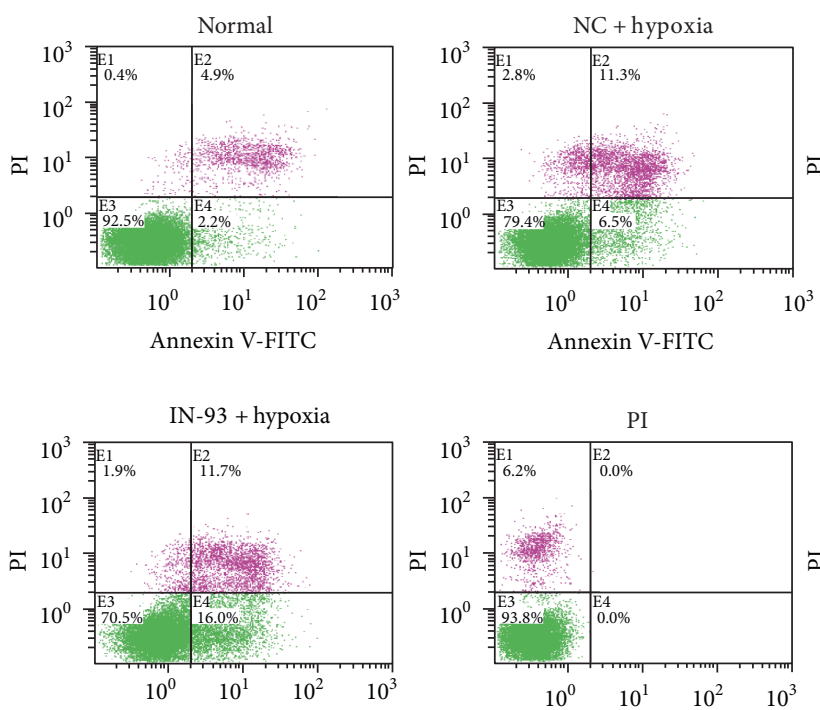

Annexin V-FITC
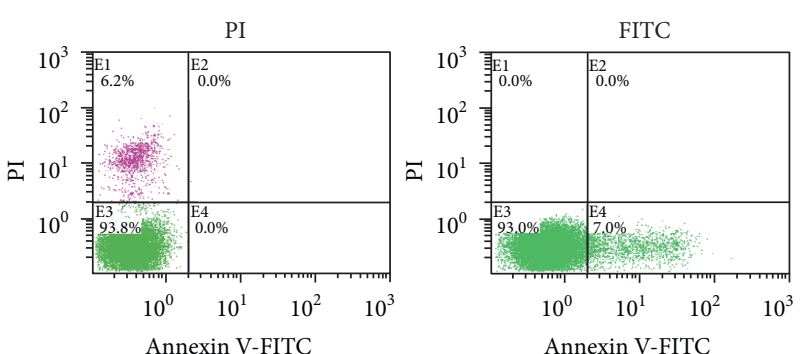

Annexin V-FITC

(c)

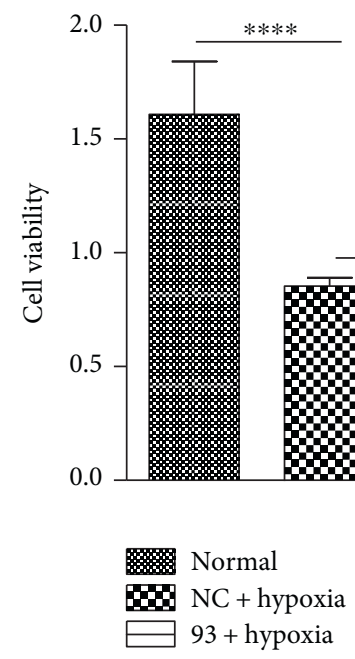

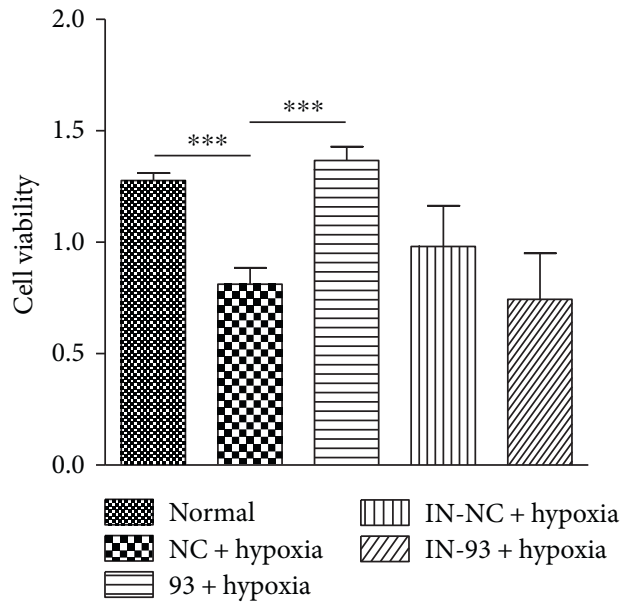

(b)
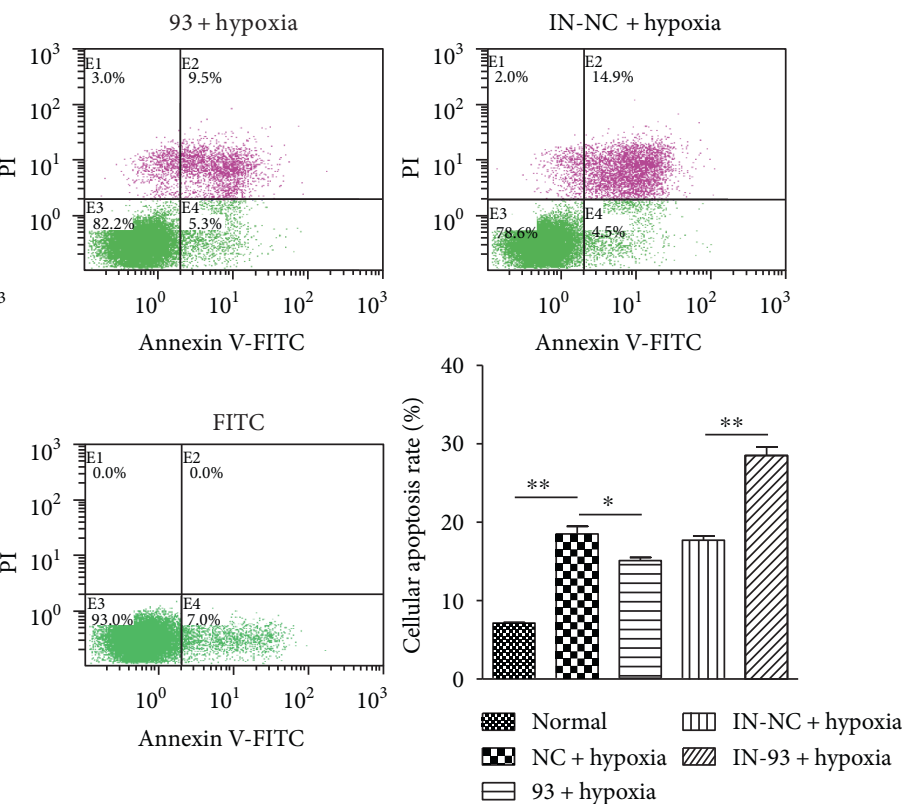


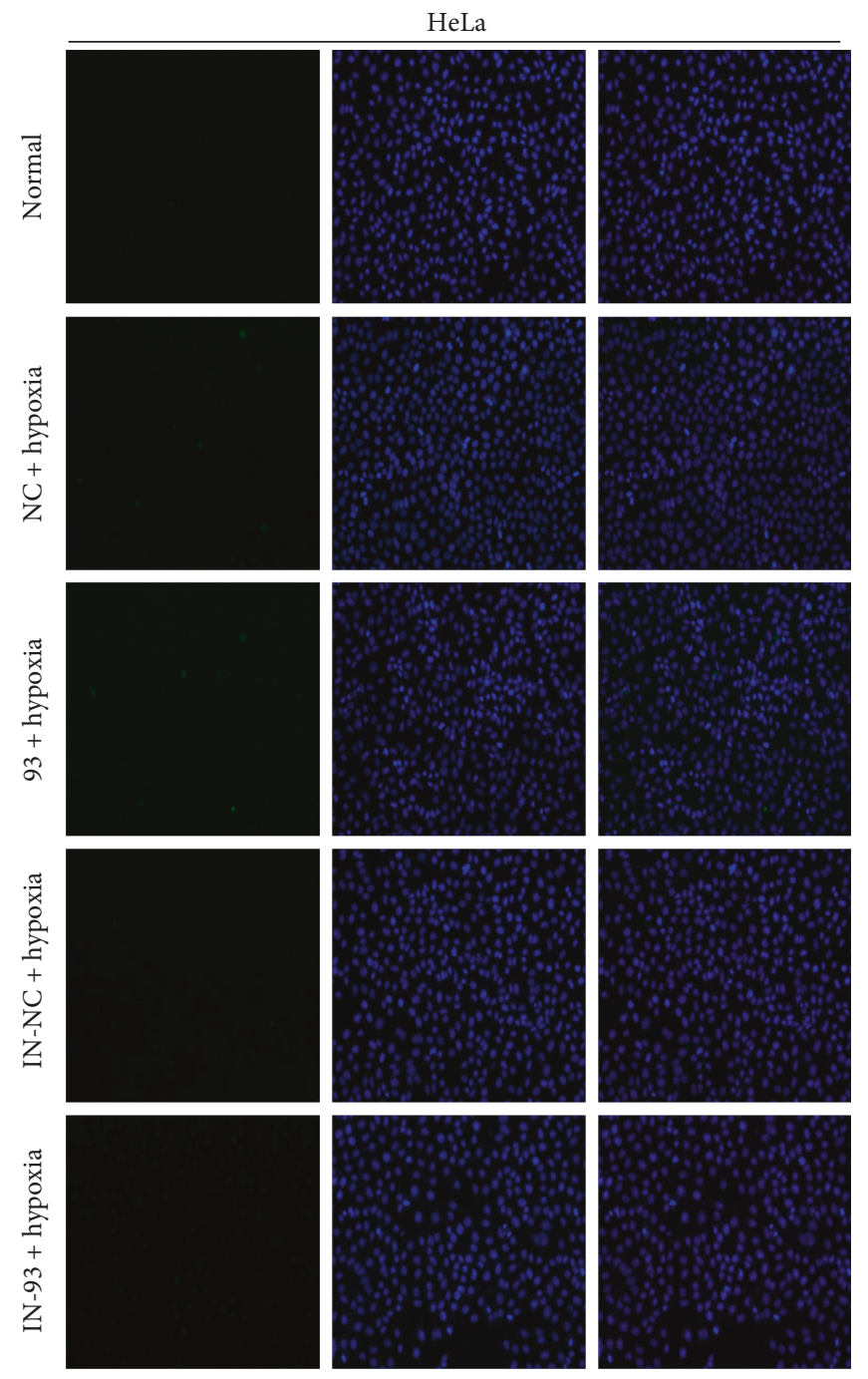

(e)

FIGURE 5: The effects of miR-93 on cell viability and apoptosis in noncancer cell lines and cancer cells. (a, b) CCK8 proliferation assays were performed on MEFs transfected with indicated RNA for $12 \mathrm{~h}$. MEFs were exposed to starvation or hypoxic conditions for another $24 \mathrm{~h}$. The graph represents the cell proliferation of three replicates with standard deviation represented by error bars. ${ }^{* *} P<0.01$; ${ }^{* * *} P<0.001$. (c) MEFs were transfected with the indicated microRNAs or left untransfected for $12 \mathrm{~h}$; the cells were exposed to hypoxic condition for another $24 \mathrm{~h}$. The cell apoptosis rate was detected by flow cytometry. Cellular apoptosis rate was calculated by GraphPad Prism 5. (d) HeLa cells were transfected with scramble NC (NC), miR-93 mimics (93), scramble IN-NC (IN-NC), or miR-93 inhibitor (IN-93) for 12 h, followed by hypoxic treatment for another $24 \mathrm{~h}$. Normal group was set as control. The CCK8 proliferation assays were detected. ${ }^{*} P<0.05$; ${ }^{* * * *} P<0.0001$. (e) HeLa cells were treated as (d), then subjected to TUNEL staining. DAPI (blue), TUNEL-positive cells (green). Magnification, 20x.

ULK1 is maintained by miR-93, and miR-93-modulated key autophagic machinery involved in hypoxic microenvironment.

\section{Conflicts of Interest}

The authors declare that they have no conflict of interests.

\section{Authors' Contributions}

$\mathrm{Du}$ Feng conceived and supervised the work. Du Feng, Liangqing Zhang, Wen Li, and Yue Yang designed the experiments. Wen Li, Yue Yang, Zhaoyu Ba, Hao Chen, and Xiaoyan Hou performed the experiments with the help of other coauthors. Du Feng, Liangqing Zhang, Wen Li, Yue Yang, Zhaoyu Ba, Hao Chen, and Xiaoyan Hou analyzed the data. Wen Li, Yue Yang, and Du Feng wrote the paper. Wen Li, Yue Yang, Zhaoyu Ba, and Shupeng Li contributed equally to this work. Wen Li, Yue Yang, Zhaoyu $\mathrm{Ba}$, Shupeng $\mathrm{Li}$ are the co-first author of the paper.

\section{Acknowledgments}

This work was supported by the NSFC (no. 31771531, no. 31401182 , no. 81270196 , and no. 81470405$)$, by the National Basic Research Program of China (2013CB910100), by the Natural Science Foundation of Guangdong Province, China 
(2014A030313533), by Yangfan Plan of Talents Recruitment Grant, Guangdong, China (Yue Cai Jiao [2015] 216), by the Science and Technology Planning Project of Guangdong Province (2016A020215152) to Du Feng, by the Key Specialty Construction Project of Shanghai Municipal Health Bureau (no. ZK2015B16) to Longxuan Li, and by the Key Discipline Construction Project of Shanghai East Hospital (YZX-2015-9).

\section{References}

[1] D. J. Klionsky, P. Codogno, A. M. Cuervo et al., “A comprehensive glossary of autophagy-related molecules and processes," Autophagy, vol. 6, pp. 438-448, 2010.

[2] D. J. Klionsky and Y. Ohsumi, "Vacuolar import of proteins and organelles from the cytoplasm," Annual Review of Cell and Developmental Biology, vol. 15, pp. 1-32, 1999.

[3] N. Mizushima, T. Yoshimori, and Y. Ohsumi, "The role of Atg proteins in autophagosome formation," Annual Review of Cell and Developmental Biology, vol. 27, pp. 107-132, 2011.

[4] N. Mizushima, B. Levine, A. M. Cuervo, and D. J. Klionsky, "Autophagy fights disease through cellular self-digestion," Nature, vol. 451, pp. 1069-1075, 2008.

[5] D. Feng, L. Liu, Y. Zhu, and Q. Chen, "Molecular signaling toward mitophagy and its physiological significance," Experimental Cell Research, vol. 319, pp. 1697-1705, 2013.

[6] J. Yan, H. Kuroyanagi, A. Kuroiwa et al., "Identification of mouse ULK1, a novel protein kinase structurally related to C. elegans UNC-51," Biochemical and Biophysical Research Communications, vol. 246, pp. 222-227, 1998.

[7] T. Hara and N. Mizushima, "Role of ULK-FIP200 complex in mammalian autophagy: FIP200, a counterpart of yeast Atg17?,” Autophagy, vol. 5, pp. 85-87, 2009.

[8] L. Shang and X. Wang, "AMPK and mTOR coordinate the regulation of Ulk1 and mammalian autophagy initiation," Autophagy, vol. 7, pp. 924-926, 2011.

[9] A. S. Loffler, S. Alers, A. M. Dieterle et al., "Ulk1-mediated phosphorylation of AMPK constitutes a negative regulatory feedback loop," Autophagy, vol. 7, pp. 696-706, 2011.

[10] D. Egan, J. Kim, R. J. Shaw, and K. L. Guan, “The autophagy initiating kinase ULK1 is regulated via opposing phosphorylation by AMPK and mTOR," Autophagy, vol. 7, pp. 643-644, 2011.

[11] L. Shang, S. Chen, F. Du, S. Li, L. Zhao, and X. Wang, "Nutrient starvation elicits an acute autophagic response mediated by Ulk1 dephosphorylation and its subsequent dissociation from AMPK," Proceedings of the National Academy of Sciences of the United States of America, vol. 108, pp. 4788-4793, 2011.

[12] J. Kim, M. Kundu, B. Viollet, and K. L. Guan, “AMPK and $\mathrm{mTOR}$ regulate autophagy through direct phosphorylation of Ulk1," Nature Cell Biology, vol. 13, pp. 132-141, 2011.

[13] J. W. Lee, S. Park, Y. Takahashi, and H. G. Wang, "The association of AMPK with ULK1 regulates autophagy," PLoS One, vol. 5, article e15394, 2010.

[14] L. R. Pike, D. C. Singleton, F. Buffa et al., "Transcriptional up-regulation of ULK1 by ATF4 contributes to cancer cell survival," The Biochemical Journal, vol. 449, pp. 389-400, 2013.

[15] W. Wu, W. Tian, Z. Hu et al., "ULK1 translocates to mitochondria and phosphorylates FUNDC1 to regulate mitophagy," EMBO Reports, vol. 15, pp. 566-575, 2014.
[16] W. Tian, W. Li, Y. Chen et al., "Phosphorylation of ULK1 by AMPK regulates translocation of ULK1 to mitochondria and mitophagy," FEBS Letters, vol. 589, pp. 1847-1854, 2015.

[17] G. Allavena, C. Boyd, K. S. Oo, E. Maellaro, B. Zhivotovsky, and V. O. Kaminskyy, "Suppressed translation and ULK1 degradation as potential mechanisms of autophagy limitation under prolonged starvation," Autophagy, vol. 12, pp. 20852097, 2016.

[18] Y. G. Zhao and H. Zhang, "ULK1 cycling: the ups and downs of the autophagy response," The Journal of Cell Biology, vol. 215, pp. 757-759, 2016.

[19] F. Nazio, M. Carinci, C. Valacca et al., "Fine-tuning of ULK1 mRNA and protein levels is required for autophagy oscillation," The Journal of Cell Biology, vol. 215, pp. 841-856, 2016.

[20] M. Hesse and C. Arenz, "MicroRNA maturation and human disease," Methods in Molecular Biology, vol. 1095, pp. 11-25, 2014.

[21] Y. Y. Mo, "MicroRNA regulatory networks and human disease," Cellular and Molecular Life Sciences, vol. 69, pp. 3529-3531, 2012.

[22] V. S. Gomase and A. N. Parundekar, "microRNA: human disease and development," International Journal of Bioinformatics Research and Applications, vol. 5, pp. 479-500, 2009.

[23] D. Gibbings, S. Mostowy, F. Jay, Y. Schwab, P. Cossart, and O. Voinnet, "Selective autophagy degrades DICER and AGO2 and regulates miRNA activity," Nature Cell Biology, vol. 14, pp. 1314-1321, 2012.

[24] S. Quenneville, P. Turelli, K. Bojkowska et al., "The KRABZFP/KAP1 system contributes to the early embryonic establishment of site-specific DNA methylation patterns maintained during development," Cell Reports, vol. 2, pp. 766-773, 2012.

[25] D. C. Schultz, K. Ayyanathan, D. Negorev, G. G. Maul, and F. J. Rauscher 3rd, "SETDB1: a novel KAP-1-associated histone $\mathrm{H} 3$, lysine 9-specific methyltransferase that contributes to HP1-mediated silencing of euchromatic genes by KRAB zinc-finger proteins," Genes \& Development, vol. 16, pp. 919932, 2002.

[26] L. Esteban-Martinez, E. Sierra-Filardi, R. S. McGreal et al., "Programmed mitophagy is essential for the glycolytic switch during cell differentiation," The EMBO Journal, vol. 36, pp. 1688-1706, 2017.

[27] W. Li, H. Chen, S. Li, G. Lin, and D. Feng, "Exploring microRNAs on NIX-dependent mitophagy," Methods in Molecular Biology, vol. 15, pp. 1-11, 2017.

[28] I. Barde, B. Rauwel, R. M. Marin-Florez et al., “A KRAB/KAP1miRNA cascade regulates erythropoiesis through stage-specific control of mitophagy," Science, vol. 340, pp. 350-353, 2013.

[29] W. Li, X. Zhang, H. Zhuang et al., "MicroRNA-137 is a novel hypoxia-responsive microRNA that inhibits mitophagy via regulation of two mitophagy receptors FUNDC1 and NIX," The Journal of Biological Chemistry, vol. 289, pp. 1069110701, 2014.

[30] F. Jin, Y. Wang, M. Li et al., "MiR-26 enhances chemosensitivity and promotes apoptosis of hepatocellular carcinoma cells through inhibiting autophagy," Cell Death \& Disease, vol. 8, article e2540, 2017.

[31] Y. Lin, W. Deng, J. Pang et al., "The microRNA-99 family modulates hepatitis $\mathrm{B}$ virus replication by promoting IGF-1R/PI3K/Akt/mTOR/ULK1 signaling-induced autophagy," Cellular Microbiology, vol. 19, no. 5, 2017. 
[32] S. I. Rothschild, O. Gautschi, J. Batliner, M. Gugger, M. F. Fey, and M. P. Tschan, "MicroRNA-106a targets autophagy and enhances sensitivity of lung cancer cells to Src inhibitors," Lung Cancer, vol. 107, pp. 73-83, 2017.

[33] S. D'Adamo, O. Alvarez-Garcia, Y. Muramatsu, F. Flamigni, and M. K. Lotz, "MicroRNA-155 suppresses autophagy in chondrocytes by modulating expression of autophagy proteins," Osteoarthritis and Cartilage, vol. 24, pp. 1082-1091, 2016.

[34] Y. Chen, S. Wang, L. Zhang et al., "Identification of ULK1 as a novel biomarker involved in miR-4487 and miR-595 regulation in neuroblastoma SH-SY5Y cell autophagy," Scientific Reports, vol. 5, article 11035, 2015.

[35] X. Duan, T. Zhang, S. Ding et al., "microRNA-17-5p modulates Bacille Calmette-Guerin growth in RAW264.7 cells by targeting ULK1," PLoS One, vol. 10, article e0138011, 2015.

[36] Z. Wang, N. Wang, P. Liu et al., "MicroRNA-25 regulates chemoresistance-associated autophagy in breast cancer cells, a process modulated by the natural autophagy inducer isoliquiritigenin," Oncotarget, vol. 5, pp. 7013-7026, 2014.

[37] H. Wu, F. Wang, S. Hu et al., "MiR-20a and miR-106b negatively regulate autophagy induced by leucine deprivation via suppression of ULK1 expression in C2C12 myoblasts," Cellular Signalling, vol. 24, pp. 2179-2186, 2012.

[38] Y. Chen, R. Liersch, and M. Detmar, “The miR-290-295 cluster suppresses autophagic cell death of melanoma cells," Scientific Reports, vol. 2, p. 808, 2012.

[39] M. Otsuka, Q. Jing, P. Georgel et al., "Hypersusceptibility to vesicular stomatitis virus infection in Dicer1-deficient mice is due to impaired miR24 and miR93 expression," Immunity, vol. 27, pp. 123-134, 2007.

[40] X. Chen, H. Yang, X. Zhou, L. Zhang, and X. Lu, "MiR-93 targeting EphA4 promotes neurite outgrowth from spinal cord neurons," Journal of Molecular Neuroscience, vol. 58, pp. 517-524, 2016.

[41] Z. P. Ke, P. Xu, Y. Shi, and A. M. Gao, "MicroRNA-93 inhibits ischemia-reperfusion induced cardiomyocyte apoptosis by targeting PTEN,” Oncotarget, vol. 7, pp. 28796-28805, 2016.

[42] L. Jiang, C. Wang, F. Lei et al., "miR-93 promotes cell proliferation in gliomas through activation of PI3K/Akt signaling pathway," Oncotarget, vol. 6, pp. 8286-8299, 2015.

[43] W. Li, Y. Yang, X. Hou et al., "MicroRNA-495 regulates starvation-induced autophagy by targeting ATG3," FEBS Letters, vol. 590, pp. 726-738, 2016.

[44] D. Papinski and C. Kraft, "Atg1 kinase organizes autophagosome formation by phosphorylating Atg9," Autophagy, vol. 10, pp. 1338-1340, 2014.

[45] D. Papinski, M. Schuschnig, W. Reiter et al., "Early steps in autophagy depend on direct phosphorylation of Atg9 by the Atg1 kinase," Molecular Cell, vol. 53, pp. 471-483, 2014.

[46] N. Agrawal, P. V. Dasaradhi, A. Mohmmed, P. Malhotra, R. K. Bhatnagar, and S. K. Mukherjee, "RNA interference: biology, mechanism, and applications," Microbiology and Molecular Biology Reviews, vol. 67, pp. 657-685, 2003.

[47] S. M. Elbashir, J. Harborth, W. Lendeckel, A. Yalcin, K. Weber, and T. Tuschl, "Duplexes of 21-nucleotide RNAs mediate RNA interference in cultured mammalian cells," Nature, vol. 411, pp. 494-498, 2001.

[48] T. Yonekawa and A. Thorburn, "Autophagy and cell death," Essays in Biochemistry, vol. 55, pp. 105-117, 2013.
[49] K. M. Chung and S. W. Yu, "Interplay between autophagy and programmed cell death in mammalian neural stem cells," $B M B$ Reports, vol. 46, pp. 383-390, 2013.

[50] R. L. Macintosh and K. M. Ryan, "Autophagy in tumour cell death," Seminars in Cancer Biology, vol. 23, pp. 344-351, 2013.

[51] Z. Zou, L. Wu, H. Ding et al., "MicroRNA-30a sensitizes tumor cells to cis-platinum via suppressing beclin 1-mediated autophagy," The Journal of Biological Chemistry, vol. 287, pp. 4148-4156, 2012.

[52] H. Zhu, H. Wu, X. Liu et al., "Regulation of autophagy by a beclin 1-targeted microRNA, miR-30a, in cancer cells," Autophagy, vol. 5, pp. 816-823, 2009.

[53] S. Comincini, G. Allavena, S. Palumbo et al., "microRNA-17 regulates the expression of ATG7 and modulates the autophagy process, improving the sensitivity to temozolomide and low-dose ionizing radiation treatments in human glioblastoma cells," Cancer Biology \& Therapy, vol. 14, pp. 574-586, 2013.

[54] K. A. Tekirdag, G. Korkmaz, D. G. Ozturk, R. Agami, and D. Gozuacik, "MIR181A regulates starvation- and rapamycininduced autophagy through targeting of ATG5," Autophagy, vol. 9, pp. 374-385, 2013.

[55] P. Zhang and H. Zhang, "Autophagy modulates miRNAmediated gene silencing and selectively degrades AIN-1/ GW182 in C. elegans," EMBO Reports, vol. 14, pp. 568-576, 2013.

[56] S. Hazarika, C. R. Farber, A. O. Dokun et al., "MicroRNA-93 controls perfusion recovery after hind-limb ischemia by modulating expression of multiple genes in the cell cycle pathway," Circulation, vol. 127, pp. 1818-1828, 2013.

[57] F. Nazio, F. Strappazzon, M. Antonioli et al., "mTOR inhibits autophagy by controlling ULK1 ubiquitylation, selfassociation and function through AMBRA1 and TRAF6," Nature Cell Biology, vol. 15, pp. 406-416, 2013. 


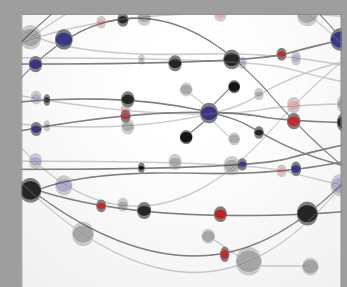

The Scientific World Journal
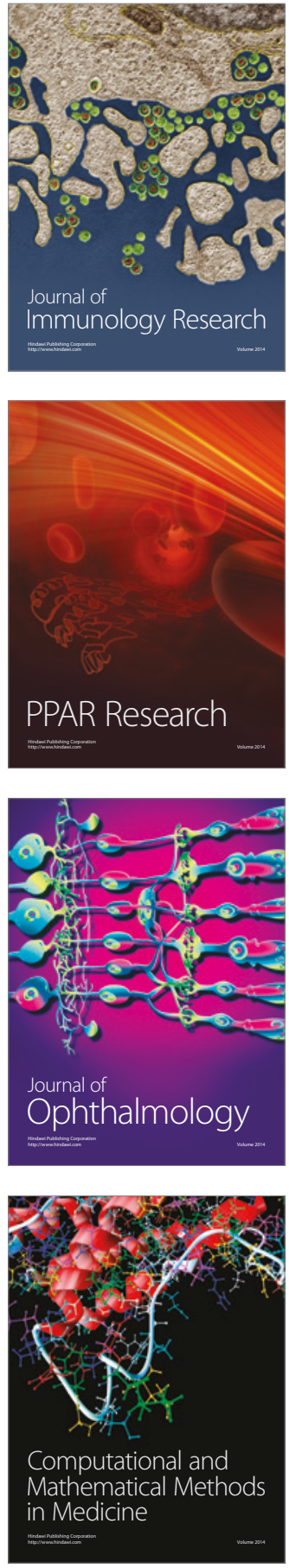

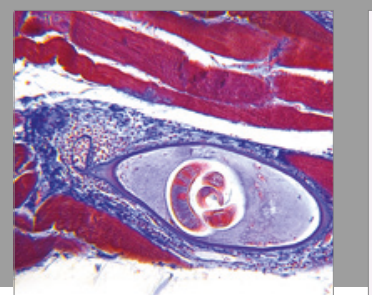

Gastroenterology Research and Practice
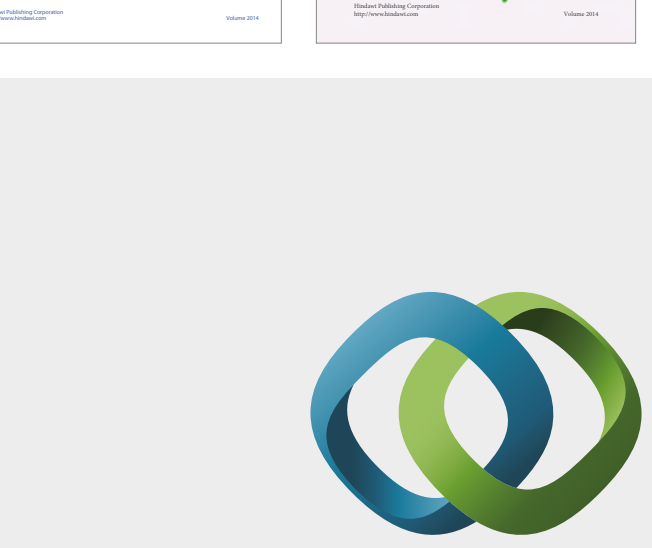

\section{Hindawi}

Submit your manuscripts at

https://www.hindawi.com
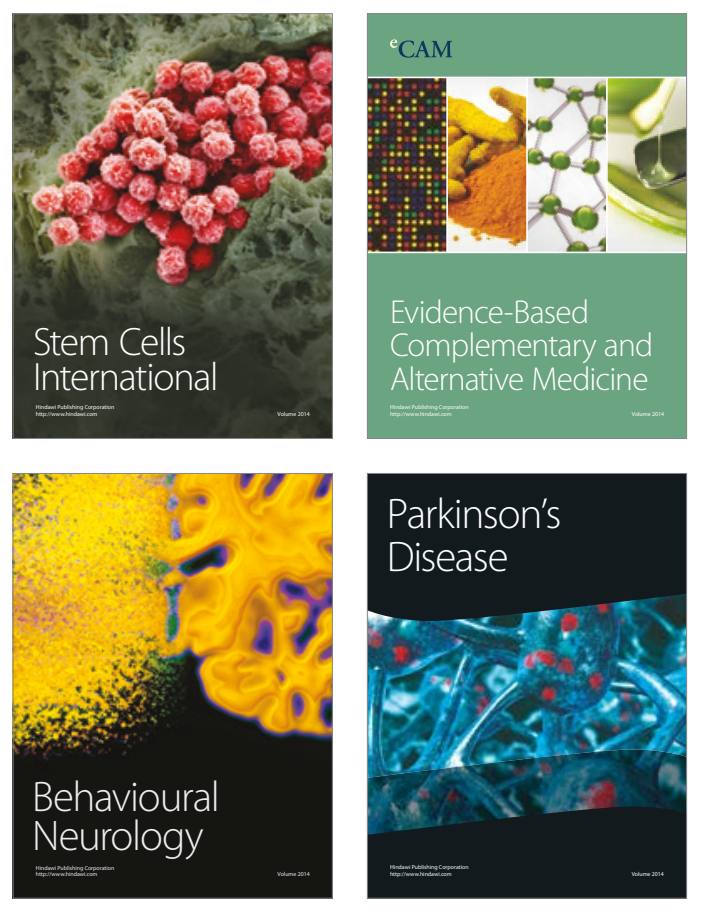
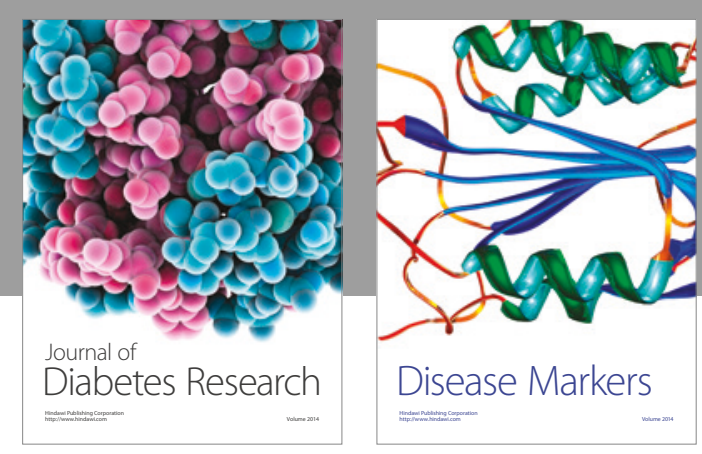

Disease Markers
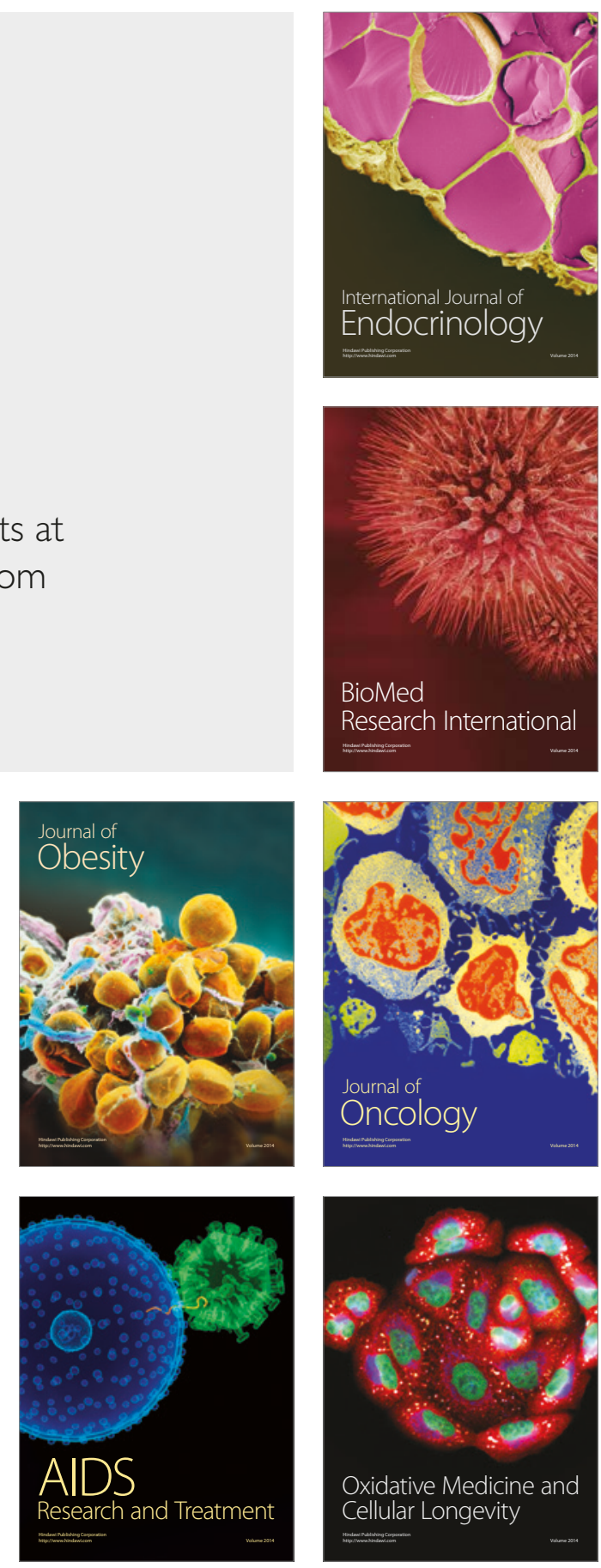